\title{
Etudes cytologiques de choux tétraploïdes (Brassica oleracea L. ssp. acephala) obtenus à partir de lignées diploïdes après traitement à la colchicine
}

\author{
A.-M. Chevre, F. Eber, G. Thomas et F. Baron \\ INRA, Station d'amélioration des Plantes, BP 29, F35650 Le Rheu, France
}

(reçu le 2 août 1988, accepté le 26 février 1989)

\begin{abstract}
Résumé - Des graines en germination de quatre lignées diploïdes de chou fourrager ont été traitées à la colchicine. Seules les plantules présentant un nombre moyen de chloroplastes par cellules stomatiques supérieur à celui des témoins ont été retenues. Un nouveau dénombrement au stade adulte a permis d'établir une bonne corrélation entre les résultats obtenus par cette méthode et le niveau de ploïdie déterminé par dénombrement chromosomique. La proportion de plantes autotétraploïdes, au sein du matériel sélectionné, dépend de la lignée d'origine. Leur comportement méiotique est de type diploïde et leur fertilité satisfaisante.
\end{abstract}

Brassica oleracea - niveau de ploïdie - chloroplastes - comportement méiotique

Summary - Cytological studies of tetraploid kale (Brassica oleracea L. ssp acephala) obtained from diploid lines after colchicine treatment. Germinating seeds for four kale diploid lines were treated by colchicine. Only the seedlings with a mean number of chloroplasts per stomatic cells superior to that of controls were selected. A new counting at adult stage has allowed us to establish a good correlation between the results of this method and the ploidy level, determined by chromosomic counting. The proportion of autotetraploid plants within the selected material depends on the line used. Their meiotic behaviour is diploid-typed and their fertility is fairly high.

Brassica oleracea - ploidy level - chloroplasts - meiosis

\section{Introduction}

Le chou, Brassica oleracea L. (CC, $2 n=18)$ est l'une des trois espèces diploïdes de la tribu des Brassiceae, ces dernières ayant donné naissance à trois allotrétraploïdes $(U, 1935)$. Aucun autotrétraploïde naturel n'existe dans ce groupe mais ils ont pu être obtenus artificiellement ou spontanément. S'ils présentent généralement une vigueur accrue, leur fertilité, estimée par le rendement en grains, est réduite du fait d'une méiose irrégulière (Prakash et Hinata, 1980). Ceci a limité l'intérêt porté à ce matériel, la plupart des espèces de cette tribu étant cultivées pour leurs graines en tant qu'oléagineux.

Chez le chou fourrager (ssp. acephala), de type bisannuel, dont seule la partie végétative est utilisée en fourrage frais, l'exploitation de la vigueur des tétraploïdes entraînant une augmen- tation du poids de matière sèche et de la teneur en protéines (Olsson et Ellerström, 1980), a conduit à la création de quelques variétés cultivées telles que Tema (Suède) et Polycaul (Fran$\mathrm{ce})$, de type variétés-populations. Cependant aucune étude cytologique n'a été réalisée; seuls quelques résultats ont été rapportés chez d'autres cultigroupes de B. oleracea (Howard, 1939; McNaughton, 1973).

Nos objectifs ont donc été de créer un matériel utilisable dans les programmes d'amélioration du chou fourrager et d'appréhender les mécanismes de régulation des appariements chromosomiques.

Par ailleurs, la méthode d'estimation du niveau de ploïdie la plus fréquemment utilisée chez $B$. oleracea, tant pour distinguer les plantes diploïdes des tétra et octoploïdes (Horàk et al., 
1975) que les plantes haploïdes des diploïdes (Doré, 1986), est la longueur des cellules de garde des stomates. Ces auteurs signalent, pour ce paramètre, l'importance du prélèvement et de l'âge de la plante. Souhaitant, pour notre part, sélectionner à différents moments de la période végétative et ayant noté une importante hétérogénéité de la taille des stomates au sein d'une même plante, nous avons cherché à transposer, chez le chou, la méthode préconisée chez la betterave (Essad et Touvin, 1959).

Après traitement à la colchicine de lignées diploïdes, nous présenterons les résultats obtenus sur l'évaluation précoce du niveau de ploïdie ainsi que sur le comportement méiotique du matériel obtenu. L'intérêt de la technique de dénombrement des chloroplastes dans les cellules de garde des stomates pour apprécier le niveau de ploïdie des plantes sera examiné et discuté.

\section{Matériel et Méthodes}

\section{Matériel}

Quatre lignées de choux diploïdes et intercompatibles ont été utilisées : C 51, CB 151, F 155 et P 57. Elles ont été extraites de quatre populations appartenant à des types fourragers différents et produites par autofécondation forcée au "stade bouton floral" pour lever l'autoincompatibilité de l'espèce (Du Crehu, 1964). Les lignées diploïdes d'origine sont utilisées comme témoins par rapport au matériel traité.

\section{Méthodes}

Le traitement à la colchicine (Desprez, comm. pers., 1987) a été appliqué à 24 graines par lignée, sauf pour l'une d'entre elles (F155). Elles ont été mises en germination à $25^{\circ} \mathrm{C}$ sur perlite humidifiée. Dès l'apparition des poils absorbants, elles ont été plongées dans une solution contenant $0,05 \%$ de colchicine (Fluka $n^{\circ} 27650$ ) et $1 \%$ de Pest Oil 7 (huile minérale Du Pont Seppic Phyto) pendant 3 heures à $25^{\circ} \mathrm{C}$. Après 3 rinçages à l'eau, elles ont été semées dans du terreau en serre.

Le dénombrement des chloroplastes dans les cellules de garde des stomates a été réalisé sur des feuilles prélevées aux étages foliaires 3 ou 4 sous l'apex. Deux prélèvements au stade plantule (stade rosette) et deux prélèvements au stade adulte (stade "premiers boutons floraux") ont été effectués. Un fragment d'épiderme de la face inférieure de la feuille est détaché et monté entre lame et lamelle dans une goutte de nitrate d'argent (1\%). Vingt à trente stomates sont observés, pour un prélèvement donné, à plusieurs emplacements sur chaque feuille et sur différentes feuilles dans le cas des plantes adultes.

Pour l'étude du comportement méiotique, les premières inflorescences sont fixées dans une solution de Carnoy (alcool : 6 vol - chloroforme : 3 vol-acide acétique : $1 \mathrm{vol}$ ) pendant 24 heures à température du laboratoire. Les étamines sont ensuite dilacérées et montées entre lame et lamelle dans une goutte de car- min acétique ferrique. Vingt à trente cellules sont observées par plante.

La fertilité de la plante est estimée d'après la viabilité du pollen, mesurée après coloration du pollen par le carmin acétique. Les cellules polliniques pleines colorées en rose constituent un indice de viabilité, alors que les cellules vides non colorées indiquent la stérilité. Deux à trois fleurs épanouies le jour même sont prélevées par plante et le pourcentage de fertilité est calculé à partir de l'observation de la viabilité de 600 à 800 grains de pollen.

\section{Résultats}

Parmi les plantes issues de graines en germination traitées à la colchicine, seules celles présentant un nombre moyen de chloroplastes par cellules stomatiques $(c p)$ très supérieur à celui des témoins diploïdes ont été conservées. Un effectif de 5 plantes par lignée a été retenu systématiquement (Fig. 1).

Un dénombrement des chloroplastes dans les cellules des plantes au stade adulte, par comparaison avec celui réalisé sur plantules, a permis de classer ces dernières en 2 groupes (Fig. 1) :

- le groupe 1, caractérisé par une diminution ou un maintien (avec une valeur identique à celle des témoins examinés au stade adulte) du nombre moyen de chloroplastes : $c p$ inférieur à 17.

- le groupe 2, présentant une augmentation ou un maintien du nombre moyen de chloroplastes; $c p$ supérieur à 17.

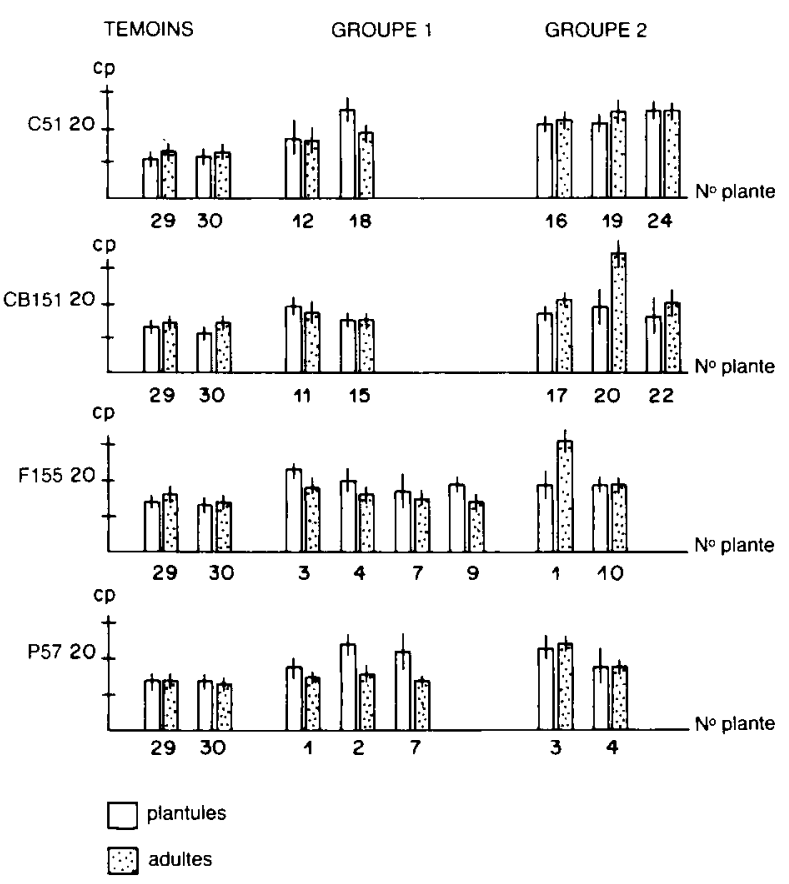

Fig. 1. Nombre moyen de chloroplastes $(c p)$ par cellules stomatiques des différentes plantes retenues. 
II est important de mentionner que l'écart-type est important (jusqu'à 5) dans le cas de certaines plantes comparées aux témoins; ceci est le reflet d'une hétérogénéité observée soit au sein d'un même fragment (ex F 155.1), soit entre feuilles prélevées sur des rameaux différents (ex CB 151.22).

L'étude du comportement méiotique a permis de révéler que l'ensemble des plantes du premier groupe, à l'exception de la plante CB 151.15, sont diploïdes $(2 n=18)$ et celles du second tétraploïdes $(2 n=36)$, à l'exception de la plante F 155.1. Dans le cas de cette dernière, des cellules diploïdes et tétraploïdes ont été observées en mélange au cours de la méiose. Les figures 2 et 3 présentent respectivement ces deux situations.

Hormis les résultats obtenus avec deux plantes, une bonne corrélation existe donc entre le nombre de chloroplastes par cellules stomatiques et le nombre de chromosomes. Cette dernière méthode permet donc d'évaluer rapidement le niveau de ploïdie.

En revanche, son efficacité pour distinguer les plantes tétraploïdes, à partir de plantules traitées,

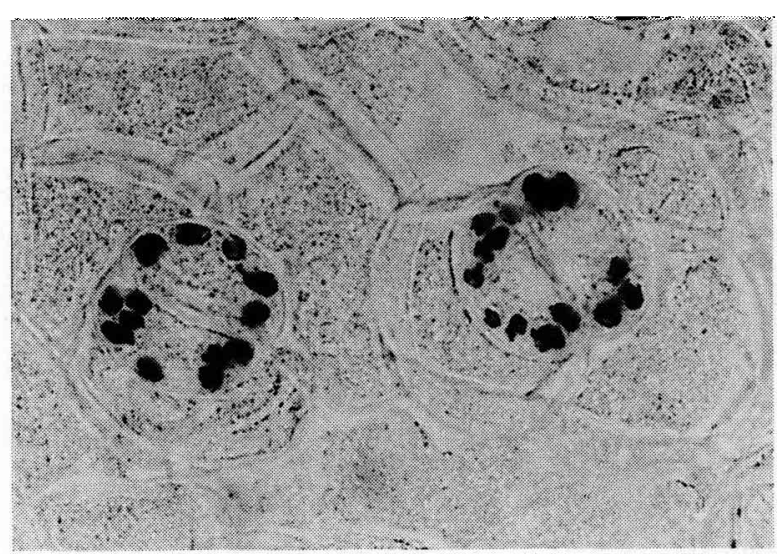

Fig. 2. Aspect des chloroplastes dans les cellules stomatiques d'une plante diploïde.

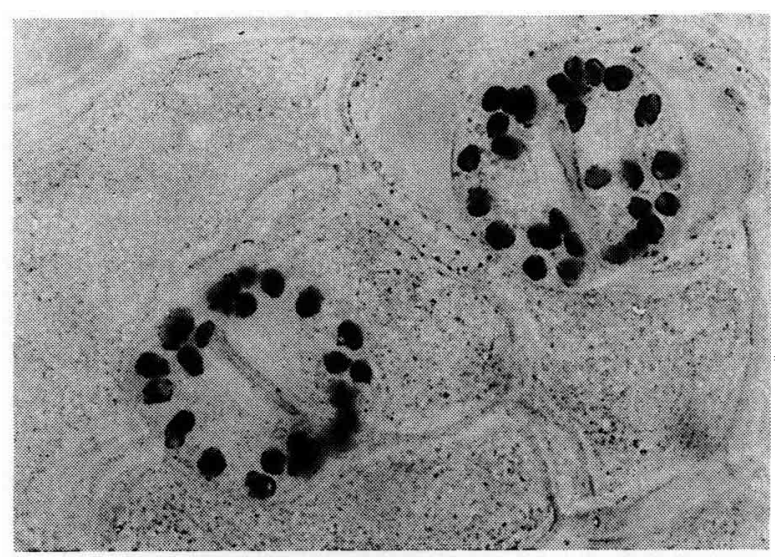

Fig. 3. Aspect des chloroplastes dans les cellules stomatiques d'une plante tétraploïde. semble fonction des lignées utilisées (Tableau I); le pourcentage de plantes tétraploïdes, par rapport aux plantules repérées d'après le nombre moyen de chloroplastes, varie de 20 à $80 \%$.

Malgré le faible pourcentage de plantes tétraploïdes obtenues (Tableau I), il nous a été possible d'en repérer au moins une au sein de chaque lignée utilisée.

Les comportements méiotiques et les fertilités polliniques sont rapportés dans le Tableau II. Pour toutes les lignées, la moyenne de multivalents est plus faible que celle d'univalents (Fig. 4). La fertilité bien que de 20 à $30 \%$ inférieure à celle des témoins demeure bonne (60 à $70 \%)$.

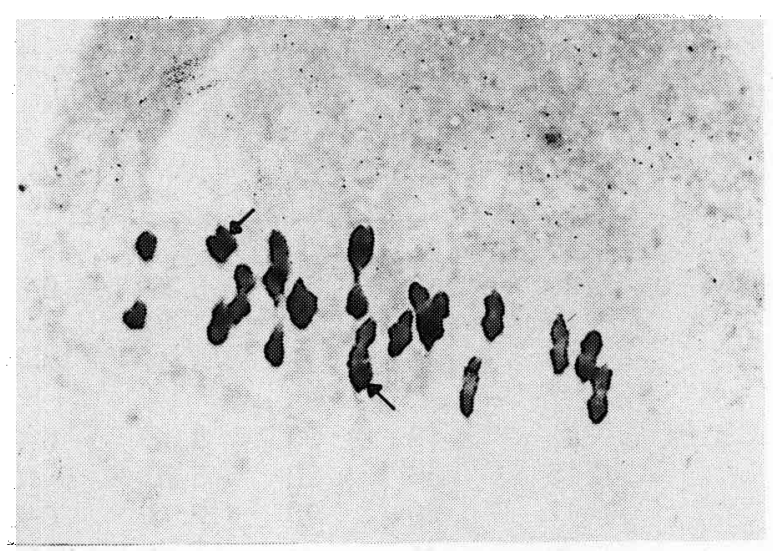

Fig. 4. Métaphase I de la méiose d'une plante tétraploïde : $17^{\prime \prime}+2^{\prime}(->)$.

\section{Discussion}

Pour cette méthode d'évaluation précoce du niveau de ploïdie, une bonne corrélation a pu être établie entre le niveau de ploïdie et le nombre de chloroplastes dans les cellules stomatiques.

Cependant, l'hétérogénéité notée au sein de certaines plantes, qui se traduit par un écart-type important, et les anomalies constatées au cours de la méiose, révèlent probablement leur nature mixoploïde. Ceci peut être rapproché des observations réalisées par Horak et al. (1975) qui suggèrent que les cellules de la tige pourraient être mixoploïdes. Par ailleurs, les tissus traités sont plus ou moins en division. Enfin une action non homogène de la colchicine est la plus probable; elle pourrait expliquer également que des plantes repérées comme tétraploïdes au stade plantule, réversent vers l'état diploïde au stade adulte par développement de parties non doublées. $\mathrm{Ce}$ phénomène a déjà été repéré chez l'asperge (Doré, 1976) montrant notamment, pour le doublement d'iiaploïdes, une compétition entre les cellules traitées pour le doublement, peu nombreuses, et le reste de la plante, haploïde. Cela 
Tableau I. Résultats obtenus après traitement à la colchicine de lignées diploïdes.

\begin{tabular}{|c|c|c|c|c|c|}
\hline \multirow[t]{2}{*}{ Lignées } & \multirow{2}{*}{$\begin{array}{l}\text { Nombre } \\
\text { de graines } \\
\text { en germination } \\
\text { traitées }\end{array}$} & \multirow{2}{*}{$\begin{array}{l}\text { Nombre } \\
\text { de plantules } \\
\text { retenues }\end{array}$} & \multirow{2}{*}{$\begin{array}{l}\text { Nombre } \\
\text { de plantes } \\
\text { adultes } \\
\text { tétraploïdes }\end{array}$} & \multicolumn{2}{|c|}{$\%$ de plantes tétraploïdes } \\
\hline & & & & $\begin{array}{l}\% \text { par rapport } \\
\text { au total }\end{array}$ & $\begin{array}{l}\text { \% par rapport } \\
\text { aux plantes } \\
\text { retenues }\end{array}$ \\
\hline $\mathrm{C} 51$ & 24 & 5 & 3 & 12,5 & 60 \\
\hline CB151 & 24 & 5 & 4 & 16,7 & 80 \\
\hline F155 & 12 & 5 & 1 & 8,3 & 20 \\
\hline P57 & 24 & 5 & 2 & 8,3 & 40 \\
\hline
\end{tabular}

Tableau II. Comportements méiotiques et fertilité pollinique des lignées diploïdes et tétraploïdes.

\begin{tabular}{|c|c|c|c|c|c|c|c|c|c|c|c|c|}
\hline \multirow[t]{2}{*}{ Lignées } & \multirow{2}{*}{$\begin{array}{l}N^{\circ} \\
\text { plante }\end{array}$} & \multirow{2}{*}{$2 n$} & \multirow{2}{*}{$\begin{array}{c}\text { Nombre } \\
\text { C }\end{array}$} & \multicolumn{2}{|c|}{ Univalents } & \multicolumn{2}{|c|}{ Bivalents } & \multicolumn{2}{|c|}{ Trivalents } & \multicolumn{2}{|c|}{ Quadrivalents } & \multirow{2}{*}{$\begin{array}{l}\% \text { de } \\
\text { fertilité }\end{array}$} \\
\hline & & & & $x$ & Ext. & $x$ & Ext. & $x$ & Ext. & $x$ & Ext. & \\
\hline \multirow[t]{3}{*}{$\mathrm{C} 51$} & 29 & 18 & 30 & 0,30 & $0-2$ & 8,83 & $8-9$ & - & - & - & - & 96,40 \\
\hline & 16 & 36 & 30 & 1,46 & $0-4$ & 17,06 & $15-18$ & - & - & 0,10 & $0-1$ & 66,70 \\
\hline & 19 & 36 & 22 & 3,27 & $0-6$ & 16,27 & $15-18$ & - & - & 0,05 & $0-1$ & 68,93 \\
\hline \multirow[t]{5}{*}{ CB151 } & 29 & 18 & 30 & 0,07 & $0-2$ & 8,97 & $8-9$ & - & - & - & - & 87,64 \\
\hline & 15 & 36 & 30 & 5,20 & $0-10$ & 15,33 & $13-18$ & - & - & 0,03 & $0-1$ & 74,00 \\
\hline & 17 & 36 & 30 & 2,93 & $0-6$ & 16,47 & $15-18$ & - & - & 0,03 & $0-1$ & 72,25 \\
\hline & 20 & 36 & 24 & 3,96 & $0-10$ & 15,54 & $13-18$ & 0,04 & $0-1$ & 0,21 & $0-1$ & 68,34 \\
\hline & 22 & 36 & 25 & 3,88 & $2-8$ & 16,00 & $14-18$ & 0,04 & $0-1$ & - & - & 62,32 \\
\hline \multirow[t]{2}{*}{ F155 } & 29 & 18 & 30 & 0,33 & $0-2$ & 8,83 & $8-9$ & - & - & - & - & 70,06 \\
\hline & 10 & 36 & 30 & 1,10 & $0-4$ & 16,93 & $15-18$ & 0,03 & $0-1$ & 0,23 & $0-1$ & - \\
\hline \multirow[t]{3}{*}{ P57 } & 29 & 18 & 30 & 0,20 & $0-2$ & 8,90 & $8-9$ & - & - & - & - & 90,19 \\
\hline & 3 & 36 & 30 & 3,86 & $2-8$ & 16,07 & $14-18$ & - & - & - & - & 70,78 \\
\hline & 4 & 36 & 30 & 1,50 & $2-6$ & 17,00 & $15-18$ & 0,03 & $0-1$ & 0,10 & $0-1$ & 68,50 \\
\hline
\end{tabular}

C: nombre de cellules observées

$X$ : moyenne

Ext. : valeurs extrêmes

aurait pour conséquence le manque d'efficacité sur certaines lignées moins sensibles à la colchicine, d'une sélection au stade plantule.

Cette difficulté, liée à une action non homogène de la colchicine, pourrait être surmontée en utilisant une autre technique de doublement. Horak et al. (1975) signalent que, par néoformation in vitro à partir de fragments de tiges de choux fourragers diploïdes, il a été possible, sans colchicine, d'obtenir $76 \%$ de tétraploïdes parmi les plantes régénérées. Le même phénomène est observé pour le doublement d'haploïdes de tabac ou de pétunia.

Lors de l'étude du comportement méiotique des témoins diploïdes, nous avons noté une faible fréquence d'univalents qui pourrait être due à une désynapsis d'un bivalent droit; en revanche aucun multivalent n'a été observé, contrairement aux résultats rapportés par Wills (1966) qui signale la présence d'un quadrivalent dans $14 \%$ des cellules analysées chez les sousespèces capitata et gemmifera. De même, chez les plantes tétraploïdes, la fréquence de quadrivalents, avec un maximum de 0,26 par cellule, est inférieure à celles rapportées chez d'autres cultigroupes de $B$. oleracea (de 4,0 à 1,5 par cellule) (Howard, 1939, et McNaughton, 1973). Ce dernier auteur mentionne, par ailleurs, une moyenne d'univalents inférieure $(0,46)$ à celle que nous avons observée, le minimum étant de 1,1 par cellule. L'hypothèse d'un effet dosage de 
gènes sur la régulation des appariements chromosomiques peut être avancée; en effet, si l'on observe des associations entre chromosomes homéologues à l'état haploïde (Armstrong \& Keller, 1982), nous avons pu noter un comportement méiotique régulier chez les plantes diploïdes, et une diminution du nombre de chiasmas par bras chez les plantes tétraploïdes. Ceci peut être rapproché des résultats présentés chez Triticum aestivum par Feldman (1966), qui attribue à l'augmentation des doses de gènes Ph1 la diminution du nombre de chiasmas et de chromosomes associés, due à une répulsion des chromosomes homologues.

Le comportement méiotique de type diploïde, observé chez les plantes tétraploïdes, permet la formation de gamètes bien équilibrés, et par là même, de pollen fertile. Nous pouvons donc espérer multiplier, par autofécondation, les lignées tétraploïdes obtenues même si le rendement en grains est faible, comme semblent le révéler les premiers essais, confirmant les résultats rapportés par McNaughton (1973).

Le matériel obtenu pourra être utilisé dans les programmes d'amélioration du chou fourrager pour l'obtention de lignées ou la création de variétés hybrides tétraploïdes.

Par ailleurs, des croisements entre moutarde noire ( $B$. nigra, BB) et ces lignées de choux diploïdes et tétraploïdes ont permis d'obtenir des hybrides de structure $\mathrm{CB}$ ou $\mathrm{CCB}$ respectivement, par culture d'ovaires fécondés. Ces plantes, après contrôle cytologique, seront rétrocroisées avec le parent récurrent diploïde chou afin d'obtenir des lignées d'addition chou-moutarde noire selon le même schéma que celui utilisé pour la création de lignées d'addition colza-moutarde noire (Jahier et al., 1989).

\section{Références}

Armstrong K.C. \& Keller, W.A. (1982) Chromosome pairing in haploids of Brassica oleracea. Can. J. Genet. Cytol., 24, 735-739
Dore C. (1976) Doublement du stock chromosomique d'haploïdes d'asperge (Asparagus officinalis L.) par culture in vitro des méristèmes en présence de colchicine. Ann. Amelior. Plantes 26(4), 647-655

Dore C. (1986) Evaluation du niveau de plö̈die des plantes d'une population de choux de Bruxelles (Brassica oleracea L. ssp gemmifera) d'origine pollinique. Agronomie, 6(9), 797-801

Du Crehu G. (1964) La biologie florale du chou fourrager; ses conséquences pour la sélection. Ann. Amélior. Plantes 14(1), 5-37

Essad S. \& Touvin H., (1959) Techniques de production et de contrôle des betteraves polyploïdes. $A n n$. Amélior. Plantes 4, 553-574

Feldman M. (1966) The effect of chromosomes 5B, 5D and $5 A$ on chromosomal pairing in Triticum aestivum. Proc. Nat. Acad. Sci. USA 55, 1447-1454

Horak J., Lustinec J., Mesicek J., Kaminek M. \& Polackova (1975) Regeneration of diploid and polyploid plants from the stem pit explants of diploid marrow stem kale (Brassica oleracea L.). Ann. Bot., 39, 571577

Howard H.W. (1939) The cytology of autotetraploid kale, Brassica oleracea. Cytologica 10, 77-87

Jahier J., Chevre A.-M., Tanguy A.M. \& Eber F. (1989) Extraction of disomic addition lines $B$. napus $-B$. nigra. Genome (sous presse)

McNaughton I.H. (1973) Synthesis and sterility of Raphanobrassica. Euphytica 22, 70-88

Olsson G. \& Ellerstrom S. (1980) Polyploidy breeding in Europe, p. 167-190. In: Brassica crops and wild allies; biology and breeding (Hinata S. \& GomezCampo C., eds) Japan Scientific Societies Press, Tokyo

Prakash S. \& Hinata K. (1980) Taxonomy, cytogenetics and origin of crop Brassica; a review. Opera Bot. 55, 157

U N. (1935) Genome analysis in Brassica with special reference to the experimental formation of $B$. napus and peculiar mode of fertilization. Japan J. Bot. 7, 389452

Wills A.B. (1966) Meiotic behaviour in the Brassiceae. Caryologia 9 (1), 103-116 